\title{
Parâmetros hematológicos e níveis de cortisol plasmático em vacas leiteiras de alta produção no Sul do Brasil
}

Romulo CAMPOS ${ }^{1}$

Luciana de Almeida

LACERDA $^{2}$

Silvia Resende TERRA²

Félix Hilário Diaz

GONZÁLEZ²

Correspondência para:

Félix H. D. González - LACVet (Laboratório deAnálises Clínicas Veterinárias), Faculdade de Veterinária da UFRGS. Av. Bento Gonçalves, 9090 - Bairro Agronomia. Porto Alegre, RS-Brasil. CEP: 91540-000

Recebido para publicação: 13/04/2005 Aprovado para publicação: 07/03/2008

\author{
1 - Departamento de Ciencia Animal, Universidad Nacional de Colombia, \\ Campus Palmira, Colombia \\ 2 - Universidade Federal do Rio Grande do Sul, Porto Alegre-RS
}

\section{Resumo}

A adaptação metabólica é um processo importante para a produção de leite em vacas de alta produção leiteira. A fisiologia das mudanças hematológicas e endócrinas durante o pós-parto é complexa e muitas respostas homeostáticas são importantes para obter o máximo da produção de leite. O perfil hematológico é uma ferramenta diagnóstica útil nos diferentes estágios do ciclo produtivo de vacas leiteiras. O objetivo deste estudo foi avaliar alguns parâmetros hematológicos em diferentes estágios do ciclo produtivo de vacas leiteiras e correlacionálos com o cortisol. Foram selecionadas 210 vacas multíparas, distribuídas por semanas: -3, -1 (antes do parto) e 2, 5, 8 e 11 (após o parto). Os parâmetros hematológicos avaliados foram hematócrito, concentração de hemoglobina, contagem total e diferencial de leucócitos. O estresse durante o periparto foi avaliado pela concentração de cortisol analisado através do método de radioinmunoensaio de fase sólida. Todos os parâmetros determinados (hematológicos e cortisol) estavam dentro do intervalo de referência para a espécie. Os maiores valores de cortisol foram observados na segunda semana após o parto. Alterações hematológicas associadas a sinais de alto estresse não foram observadas no presente estudo e isto provavelmente se deve ao estado de saúde satisfatório dos animais durante o período de experimento e às condições adequadas de manejo e nutrição.

\section{Introdução}

Diversos estudos propõem o acompanhamento de parâmetros hematológicos para monitorar a saúde do rebanho leiteiro e relatam a associação destes com diferentes patologias. Além disso, diferentes pesquisas relacionam problemas de imunidade com altos níveis de cortisol nas vacas. ${ }^{1}$ Várias alterações nos parâmetros hematológicos podem ser observadas durante a gestação em diferentes espécies animais e fazem parte da adaptação da fêmea ao período reprodutivo em que ela se encontra. ${ }^{2}$

No final da gestação o número de eritrócitos aumenta em conseqüência do efeito eritropoiético da somatotropina coriônica placentária, da progesterona e, talvez, da prolactina. ${ }^{3} \mathrm{O}$ volume sangüíneo materno também aumenta em resposta à circulação utero-placentária e ao desenvolvimento do feto, mantendo adequada a oxigenação dos tecidos e pressões sangüíneas, materna e fetal. ${ }^{4}$ O acompanhamento de parâmetros hematológicos em bovinos e o estudo da sua relação com outros parâmetros sangüíneos, como o cortisol, são métodos que podem ser utilizados para avaliar a resposta do organismo frente aos processos fisiológicos de cada fase do ciclo produtivo em vacas leiteiras. ${ }^{5}$

As alterações no leucograma de 
bovinos observadas durante o parto são típicas de uma resposta leucocitária a um quadro de estresse. ${ }^{6} \mathrm{O}$ número de leucócitos totais encontra-se significativamente aumentado, principalmente devido ao aumento de neutrófilos, com ou sem desvio à esquerda. O número de cada tipo de leucócito varia, dependendo do grau de estresse e da condição das membranas fetais. Estas alterações são evidentes entre as 12 e 24 horas após o parto e diminuem nos dias subseqüentes.

O número de eritrócitos totais, a concentração de hemoglobina e o hematócrito podem sofrer alterações similares durante o parto. ${ }^{2} \mathrm{Um}$ aumento significativo dos parâmetros eritrocitários foi observado durante o parto, fato relacionado com a diminuição do consumo de água durante o periparto. ${ }^{7}$ As alterações hematológicas relacionadas à lactação podem variar entre rebanhos e os baixos parâmetros eritrocitários durante este período podem estar relacionados com o nível de produção leiteira, com a nutrição do animal ${ }^{8}$ e com o aumento do volume plasmático ${ }^{9}$. Em geral, quanto maior é a produção leiteira, menores são os valores dos parâmetros eritrocitários. ${ }^{2}$

Diversos estudos indicam que a alta incidência de doenças durante e após o período de transição está ligada à imunossupressão associada ao estresse, comum em vacas leiteiras. Alterações no sistema imune de vacas leiteiras durante o periparto e início da lactação foram relatadas por diversos pesquisadores, e com o manejo cada vez mais intensificado em rebanhos leiteiros, o risco de distúrbios metabólicos pode aumentar. ${ }^{10,11}$

As alterações metabólicas ocorrem geralmente no início da lactação e são freqüentemente associadas a mudanças no manejo, na alimentação e na composição da dieta, mas também com alterações dos níveis hormonais que ocorrem durante o periparto. As alterações nos constituintes celulares e bioquímicos do sangue durante a gestação, parto e lactação em vacas leiteiras estão intimamente relacionadas com o cortisol, o hormônio do estresse. ${ }^{1,12}$
Uma resposta imune eficiente se baseia na interação e no equilíbrio entre os diferentes tipos celulares e seus produtos. A incidência de doenças infecciosas, principalmente mastite e endometrite, pode também aumentar devido à supressão das funções imunológicas durante o periparto. Com a aproximação do parto, o número de leucócitos totais aumenta, principalmente em decorrência da neutrofilia. Todavia, as capacidades funcionais destas células estão prejudicadas durante este período.

Um dos pontos-chave para o controle de uma infecção é a rápida migração e recrutamento dos neutrófilos para o local da infecção. ${ }^{13}$ Estudos recentes têm se voltado para o mecanismo pelo qual o estresse se relaciona à redução da função imunológica. Muitos autores observaram que os glicocorticóides aumentam durante o periparto e que estes são capazes de reduzir a expressão de algumas moléculas (como a L-selectina) pelos neutrófilos, o que diminui sua capacidade de migração e chegada ao local de infecção. ${ }^{1,11,14}$

Com a aproximação do parto, a quantidade de linfócitos e sua capacidade funcional diminuem, alcançando o ponto mínimo dias antes do parto. ${ }^{15}$ As células $\mathrm{T}$ são a subpopulação de linfócitos predominante na circulação sangüínea periférica dos ruminantes, chegando a $80 \%$ dos linfócitos totais. ${ }^{16}$ Algumas funções desta subpopulação de linfócitos também se alteram durante este período, como a produção de interleucinas e interferon gama. ${ }^{11}$

O objetivo deste estudo foi avaliar alguns parâmetros hematológicos como indicadores da saúde de vacas leiteiras e sua possível relação com o cortisol durante o período de transição e até a $11^{a}$ semana do ciclo produtivo de vacas de alta produção.

\section{Material e Método}

Foram selecionadas 210 vacas multíparas da raça Holandesa, com produção superior a $25 \mathrm{~kg} / \mathrm{vaca} / \mathrm{dia}$, pertencentes a cinco rebanhos comerciais considerados 
como sistemas intensivos de semiconfinamento de leite para as condições do Sul do Brasil (alimentação com base em suplementação e produção de leite acima de 8000 kilos por lactação). Após o exame clínico e verificação das condições exigidas para o estudo, foram analisadas amostras de 173 animais. A alimentação consistiu de silagens de milho e sorgo, forragem Tifton (Cynodon nlemfuensis), concentrado e suplemento mineral. Para o cálculo do valor nutritivo do alimento foi usado o programa Spartan Ration Evaluator. ${ }^{17}$

O ecossistema geográfico de localização dos rebanhos correspondeu ao planalto médio do Estado do Rio Grande do Sul. Foram avaliados dois períodos climáticos extremos para a zona temperada: inverno (dois rebanhos) e verão (três rebanhos). Em cada rebanho, os animais foram divididos em seis grupos de sete animais segundo o período do ciclo produtivo que se encontravam: pré-parto (3 e 2 semanas antes do parto esperado) e pósparto nas semanas 2, 5, 8 e 11 da lactação. As amostras do período pré-parto e as duas primeiras da lactação corresponderam ao período de transição. Com o objetivo de reduzir os efeitos de fontes de variação externas e para avaliar com maior precisão o efeito período (pre e pós parto) sobre os resultados das análises, a amostragem dos animais foi efetuada no mesmo dia em cada núcleo de produção.

Todas as amostras de sangue foram obtidas mediante punção da veia coccígea em tubos a vácuo de $5 \mathrm{~mL}$ contendo solução do ácido etilenodiaminotetra-acético tripotássico (EDTA-K ${ }_{3}$ a 10\%, como anticoagulante para a realização dos parâmetros hematológicos, e em tubos de $10 \mathrm{~mL}$ com heparina sódica para a dosagem do cortisol plasmático. Previamente a cada coleta de sangue, realizou-se o exame clínico para descartar qualquer animal doente ou suspeito de doença (problemas podais, endometrite, mastite, cetose e acidose clínica). Igualmente, foi verificado o período do qual o animal fazia parte e registrada a produção do leite dos dias prévios e do dia da coleta.
Imediatamente após a coleta de sangue, foi realizado o esfregaço sangüíneo e as lâminas foram armazenadas em recipientes adequados, protegidas da luz e de contaminação. Para a coloração do material, foi utilizado o corante de Wright (Merck, Darmstadt, Alemanha). A contagem total de leucócitos foi realizada manualmente pela técnica de contagem em câmara de Neubauer, o hematócrito foi determinado pela técnica de microhematócrito $(10.000$ rpm por 5 minutos) e a determinação da hemoglobina foi feita mediante a técnica colorimétrica do cianeto de potássio (Labtest, Lagoa Santa, MG).

A partir do esfregaço sangüíneo corado de cada animal, foi realizada a contagem diferencial de leucócitos e avaliação da morfologia celular. A determinação do cortisol plasmático foi efetuada mediante radioinmunoensaio (RIE) de fase sólida com reativos comerciais (ICN Biomedicals, Irvine CA, USA). No intuito de avaliar o estado de saúde da glândula mamária, a contagem das células somáticas (CCS) presentes no leite das vacas durante os períodos $2,5,8$ e 11 da lactação foram determinadas mediante citometria de fluxo (Somacount 300, Bentley Instruments Inc, Chaska, MN, USA).

Foram analisados os possíveis efeitos principais de rebanho e período de coleta mediante analise de variância da sub-rotina GLM do pacote estatístico SAS V8 (SAS) ${ }^{18}$ e, sempre que necessário, as variáveis foram transformadas para garantir a sua normalidade. A variável época foi analisada através do teste de $T$ para amostras independentes. Quando necessário para o teste de médias foi empregado o teste de Tukey. Igualmente, através da correlação de Pearson, foram analisadas as relações entre as variáveis sangüíneas e os valores plasmáticos do cortisol.

\section{Resultados e Discussão}

Na tabela 1 estão descritos os valores médios dos parâmetros hematológicos avaliados para os dois períodos climáticos de estudo, demonstrando que as condições 
Tabela 1 - Valores médios de variáveis hematológicas em vacas da raça Holandesa durante o verão e o inverno. Planalto Central do Rio Grande do Sul (Brasil) - período entre 2003 e 2004

\begin{tabular}{lcccccc}
\hline \multirow{2}{*}{ Variável (unidade) } & \multicolumn{3}{c}{ Inverno (n=106) } & \multicolumn{3}{c}{ Verão (n=67) } \\
\cline { 2 - 7 } Hematócrito $(\%)$ & Média & $\mathrm{DP}$ & $\mathrm{CV}$ & Média & $\mathrm{DP}$ & $\mathrm{CV}$ \\
Hemoglobina $(\mathrm{g} / \mathrm{dL})$ & $28,67^{\mathrm{a}}$ & 3,8 & 13,25 & $32,43^{\mathrm{b}}$ & 3,61 & 11,13 \\
Leucócitos totais $(/ \mu \mathrm{L})$ & $10,74^{\mathrm{a}}$ & 1,7 & 15,83 & $11,93^{\mathrm{b}}$ & 1,41 & 11,82 \\
Neutrófilos segmentados $(/ \mu \mathrm{L})$ & $5851,27^{\mathrm{a}}$ & 4891,4 & 83,60 & $10351,34^{\mathrm{b}}$ & 5565,4 & 53,77 \\
Eosinófilos $(/ \mu \mathrm{L})$ & $2708,42^{\mathrm{a}}$ & 1280,95 & 47,30 & $3497,31^{\mathrm{b}}$ & 1843,38 & 52,71 \\
Basófilos $(/ \mu \mathrm{L})$ & $572,48^{\mathrm{a}}$ & 496,35 & 86,70 & $318,49^{\mathrm{b}}$ & 352,72 & 110,75 \\
Monócitos $(/ \mu \mathrm{L})$ & $49,41^{\mathrm{a}}$ & 63,18 & 127,87 & $76,28^{\mathrm{a}}$ & 118,36 & 155,17 \\
Linfócitos $(/ \mu \mathrm{L})$ & $624,88^{\mathrm{a}}$ & 319,11 & 51,07 & $559,97^{\mathrm{a}}$ & 363,98 & 65,00 \\
Relação Neutrófilo/ Linfócito $(\mathrm{N} / \mathrm{L})$ & $0,759^{\mathrm{a}}$ & 0,49 & & $0,776^{\mathrm{a}}$ & 0,61 & 75,07 \\
\hline
\end{tabular}

Variáveis com letras diferentes nos valores da linha são estatisticamente diferentes $(\mathrm{p}<0,05)$. $\mathrm{DP}=$ desvio padrão; $\mathrm{CV}=$ coeficiente de variação

de verão influenciaram a dinâmica sangüínea na maioria dos parâmetros estudados. Esta observação já foi descrita por outros pesquisadores que encontraram variações sazonais nas contagens de leucócitos totais em bovinos, sendo os maiores observados durante o verão. ${ }^{19}$

As diferenças entre os rebanhos avaliados ocasionaram a maior variação sobre as variáveis estudadas, situação que pode ser explicada pelas alterações genéticas, de manejo e alimentação, e nos planos de sanidade que são utilizados em cada um dos rebanhos. A seleção genética para o aumento da produção de leite aumenta sensivelmente a carga metabólica das vacas leiteiras, o balanço energético tende a ser negativo durante a fase de lactação estudada o qual leva ao estresse nutricional. Estes fatores estão relacionados ao aumento da incidência de doenças infecciosas. ${ }^{11} \mathrm{~A}$ influência da dieta sobre o hemograma de bovinos também tem sido estudada, sendo observada leucocitose e monocitose em vacas alimentadas com silagem quando comparadas a vacas alimentadas com forragem verde. ${ }^{20}$

Os parâmetros hematológicos estudados encontram-se dentro dos valores de referência para a espécie bovina, e podem ser válidos para as condições ecológicas e de produção animal da região Sul e Sudeste do Brasil, onde se encontram os estados com maior produção leiteira individual do país. Em geral, a resposta leucocitária em bovinos difere daquela observada em outras espécies animais. As alterações hematológicas nesta espécie são sugestivas de considerações diagnósticas, como no caso de septicemias ou endotoxemias, sendo raramente específicas para uma determinada afecção. ${ }^{5}$ Uma septicemia bacteriana aguda freqüentemente é caracterizada por neutropenia ao invés de neutrofilia, e em ruminantes existe uma tendência menor para o desenvolvimento de uma neutrofilia significante. ${ }^{21}$

As contagens diferenciais de leucócitos (Tabela 2) obtidas neste estudo não mostraram alterações significativas. As maiores alterações hematológicas foram observadas no final da gestação, período no qual o crescimento do feto obriga uma redistribuição dos componentes sangüíneos. Observações similares já foram relatadas nas contagens totais de leucócitos em vacas durante o periparto. ${ }^{22,23}$ Outros estudos indicam que há um aumento gradual de leucócitos à medida que o parto se aproxima, observando-se um pico de aproximadamente $13 \times 10^{3} / \mathrm{mL}$ nove horas após o parto, voltando aos valores normais 3 dias após o parto. ${ }^{5}$

Normalmente, diferentes protocolos de imunização para doenças de controle não obrigatório (ex. leucose, diarréia viral bovina, rinotraqueíte bovina infecciosa, e/ou diferentes programas de controle de parasitas) são adotados para cada rebanho. É possível que estes rebanhos apresentem alterações nas variáveis hematológicas principalmente no que se refere às células 
Tabela 2 - Variáveis hematológicas segundo o período fisiológico e de lactação em vacas leiteiras de alta produção. Planalto Central do Rio Grande do Sul (Brasil) - período entre 2003 e 2004

\begin{tabular}{lcccccc}
\hline \multirow{2}{*}{ Variável (unidade) } & \multicolumn{7}{c}{ Semana(s) em relação ao parto } \\
\cline { 2 - 7 } & -3 & -1 & 2 & 5 & 8 & 11 \\
\hline Hematócrito $(\%)$ & $32,72^{\mathrm{a}}$ & $31,87^{\mathrm{a}}$ & $29,24^{\mathrm{b}}$ & $29,21^{\mathrm{b}}$ & $29,08^{\mathrm{b}}$ & $28,90^{\mathrm{b}}$ \\
Hemoglobina $(\mathrm{g} / \mathrm{dL})$ & $12,70^{\mathrm{a}}$ & $11,95^{\mathrm{a}}$ & $10,72^{\mathrm{b}}$ & $10,62^{\mathrm{b}}$ & $10,88^{\mathrm{b}}$ & $10,54^{\mathrm{b}}$ \\
Leucócitos totais $(/ \mu \mathrm{L})$ & 7011,60 & 8080,81 & $90,44,47$ & 8414,22 & 6684,60 & 6348,17 \\
Neutrófilos segmentados $(/ \mu \mathrm{L})$ & 2566,9 & 3187,9 & 3198,73 & 3551,36 & 2725,78 & 2724,46 \\
Eosinófilos $(/ \mu \mathrm{L})$ & $750,69^{\mathrm{a}}$ & $578,97^{\mathrm{a}, \mathrm{b}}$ & $438,31^{\mathrm{b}}$ & $368,16^{\mathrm{b}}$ & $366,27^{\mathrm{b}}$ & $366,98^{\mathrm{b}}$ \\
Basófilos $(/ \mu \mathrm{L})$ & 65,89 & 65,85 & 46,34 & 78,41 & 3190 & 68,62 \\
Monócitos $(/ \mu \mathrm{L})$ & 563,59 & 569,12 & 658,16 & 680,50 & 629,94 & 494,01 \\
Linfócitos $(/ \mu \mathrm{L})$ & 5458,79 & 5505,45 & 5396,52 & 5198,4 & 4659,18 & 4370,01 \\
Relação N/L & 0,6073 & 0,7117 & 0,797 & 1,0086 & 0,7733 & 0,6766 \\
\hline Vaívis com
\end{tabular}

Variáveis com letras diferentes nos valores da linha são estatisticamente diferentes $(p<0,05)$

associadas à defesa celular (ex. linfócitos T). No presente estudo, observou-se que o rebanho influenciou de forma contundente a resposta hematológica, quando comparado ao efeito período (momento da coleta).

O cortisol plasmático foi monitorado para avaliar o nível de estresse durante o periparto. O comportamento deste hormônio nos diferentes períodos pode ser observado na figura 1 . Os maiores valores foram detectados na segunda semana após o parto, situação que é fisiologicamente aceitável, uma vez que neste período a produção do leite é desencadeada e há aumento da síntese de lactose para garantir a maior produção de leite e outros produtos da gliconeogênese modulada pelo cortisol. Valores altos de cortisol também foram encontrados durante o pré-parto, o que também pode estar relacionado com o período de transição, caracterizado por grandes desafios na homeostasia. O cortisol não foi dosado no dia do parto, mas outros autores encontraram elevados valores neste dia. ${ }^{1}$

Muitas alterações endócrinas no final da gestação e início da lactação são devidas a mudanças fisiológicas que ocorrem neste período e ao aumento dos nutrientes requeridos para a lactação. ${ }^{24}$ As alterações

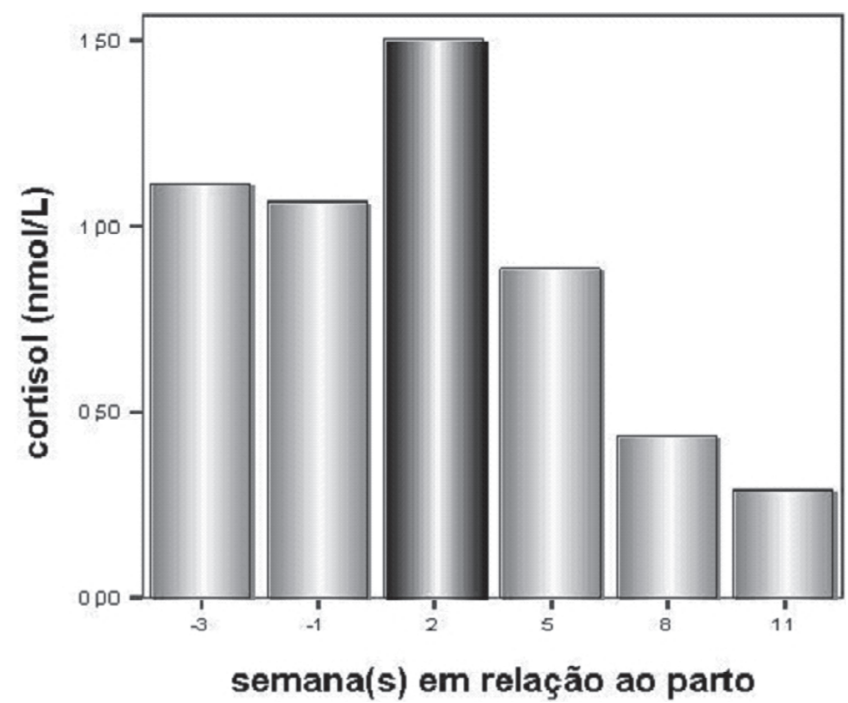

Figura 1 - Valores plasmáticos de cortisol (nmol/L) durante o período produtivo em vacas leiteiras de alta produção no Planalto Central do Rio Grande do Sul (Brasil) - período entre 2003 e 2004 [valores: - 3 sem - 1,1170 $\pm 0,98 \mathrm{nmol} / \mathrm{L} ;-1 \mathrm{sem}-1,0654 \pm 0,95 \mathrm{nmol} / \mathrm{L} ; 2 \mathrm{sem}-1,5033 \pm 1,26 \mathrm{nmol} / \mathrm{L} ; 5 \mathrm{sem}-0,8851 \pm 1,24$ $\mathrm{nmol} / \mathrm{L} ; 8 \mathrm{sem}-0,4348 \pm 1,06 \mathrm{nmol} / \mathrm{L} ; 11 \mathrm{sem}-0,2924 \pm 1,00 \mathrm{nmol} / \mathrm{L}]$ 
hormonais e metabólicas podem, direta ou indiretamente, comprometer a imunocompetência do animal e levar a um aumento da susceptibilidade a infecções bacterianas durante esta fase. ${ }^{25}$

Os leucócitos, essenciais componentes do sistema imune, expressam receptores funcionais para muitos hormônios (cortisol, insulina, leptina) que atuam mediando a homeorrese e a homeostase na vaca leiteira. ${ }^{11}$ Os níveis destes hormônios se alteram drasticamente durante o período do periparto, sendo responsáveis em parte pela produção leiteira e pela mobilização do tecido corporal. O sistema endócrino gera uma mudança metabólica coordenada para suporte do período do parto e da lactação e isso resulta em alterações marcantes em um grande número de metabólitos. Estas mudanças metabólicas podem afetar diversos órgãos e sistemas, entre eles o sistema imune. Por exemplo, a infiltração hepática de lipídios pode afetar a produção de proteínas de fase aguda, além de afetar a função das células do sistema imune e a função leucocitária. Estas alterações causam uma diminuição da habilidade bactericida destas células, o que tem sido associado com a alta incidência e severidade da mastite. ${ }^{26,27}$

$\mathrm{Na}$ tabela 3, estão apresentadas as correlações entre o cortisol e as variáveis hemáticas. Como esperado, houve uma relação significativa entre hematócrito e hemoglobina e entre linfócitos e leucócitos, monócitos e hematócrito.

Como foi observado no presente estudo, níveis superiores de cortisol plasmático próximo ao parto poderiam estar relacionados com uma depressão do sistema imune. Vários autores relatam que altos níveis de cortisol estão relacionados com a diminuição dos níveis de L-selectina ${ }^{22}$ e menor secreção de proteínas, que são essenciais para uma resposta imunológica normal, celular e humoral ${ }^{11}$.

As células somáticas do leite são, em sua maioria, células de defesa, principalmente leucócitos, que migram do sangue para o interior da glândula mamária com o objetivo de combater agentes patogênicos, e em menor grau células epiteliais, provenientes do processo de descamação do tecido da glândula mamária. A contagem de células somáticas (CCS) do leite indica o grau de infecção da glândula mamária. ${ }^{28}$ No presente estudo, não houve correlação significativa entre os diferentes tipos de células sangüíneas e a CCS durante os períodos de lactação considerados, isto provavelmente foi devido ao manejo da ordenha e à ausência de mastite, avaliados durante a seleção dos animais, e ao baixo número de células somáticas.

\section{Conclusões}

Não foram encontradas correlações significativas entre o cortisol plasmático e a maioria dos parâmetros hematológicos avaliados. Os resultados indicam que os animais do estudo estão sob condições de saúde e manejo satisfatórias, uma vez que não há indício de estresse clínico. A maior concentração de cortisol foi encontrada durante a segunda semana de lactação. Além

Tabela 3 - Correlações entre o cortisol e as variáveis hematológicas em vacas leiteiras de alta produção. Planalto Central do Rio Grande do Sul (Brasil) - período entre 2003 e 2004

\begin{tabular}{|c|c|c|c|c|c|c|c|c|c|c|}
\hline & Cortisol & HT & $\mathrm{HB}$ & LT & $\mathrm{NB}$ & NS & EOS & BAS & MON & LIN \\
\hline Cortisol & 1 & & & & & & & & & \\
\hline HT & 0,0171 & 1 & & & & & & & & \\
\hline $\mathrm{Hb}$ & $-0,024$ & $0,741 * *$ & 1 & & & & & & & \\
\hline LT & 0,074 & $0,196^{*}$ & 0,147 & 1 & & & & & & \\
\hline NB & 0,034 & 0,031 & $-0,007$ & 0,039 & 1 & & & & & \\
\hline NS & $-0,092$ & 0,089 & 0,056 & $0,453 * *$ & $0,248 * *$ & 1 & & & & \\
\hline EOS & $-0,109$ & 0,068 & 0,155 & 0,118 & $-0,077$ & 0,006 & 1 & & & \\
\hline BAS & 0,041 & 0,009 & 0,076 & 0,021 & 0,031 & $-0,027$ & 0,087 & 1 & & \\
\hline $\mathrm{MON}$ & 0,126 & 0,120 & $-0,128$ & $0,391 * *$ & $-0,032$ & $0,221 * *$ & $-0,037$ & 0,010 & 1 & \\
\hline LIN & $-0,046$ & $0,190^{*}$ & 0,136 & $0,913 * *$ & 0,072 & 0,074 & 0,012 & 0,000 & $0,273 * *$ & 1 \\
\hline
\end{tabular}


disso, não houve correlação significativa entre os valores hematológicos e a CCS do leite. Os valores hematológicos obtidos neste estudo podem ser usados como valores de referência para bovinos de raça Holandesa sob condições de produção do
Sul do Brasil. O estudo de métodos de auxílio diagnóstico para uma avaliação geral do rebanho, como a determinação de alguns parâmetros hematológicos, permite uma oportuna ação sobre o manejo e saúde dos animais.

\title{
Hematological parameters and plasma cortisol levels in high-yielding dairy cows in the South of Brazil
}

\begin{abstract}
The metabolic adaptation is an important process to milk production in high-yielding dairy cows. The physiology of hematological and endocrinal changes during the postpartum period is complex and many homeostatic responses are important to achieve the maximum milk production. The hematological status is an useful diagnostic tool to evaluate the general health and the immune system on different stages of the productive cycle in dairy cows. The objective of this study was to evaluate some hematological parameters in different periods of the productive cycle of dairy cows and correlate them with cortisol. Two hundred and ten multiparous dairy cows were selected to this experiment. The animals were distributed by weeks: -3, -1 (60 and 15 days before calving), 2, 5, 8 and 11 (weeks postpartum). The hematological parameters analyzed were PCV (packed cell volume), hemoglobin concentration, total and differential count of WBC (white blood cells). The stress during the periparturient period was evaluated by cortisol levels analyzed by solid-phase radioimmunoassay method. All the hematological and cortisol values were within the normal range for the species. The highest values for cortisol were found during the second week. Hematological changes associated to high stress signs were not observed in the present study and this is probably due to the satisfactory health state during the experiment period and the adequate nutritional and management conditions.
\end{abstract}

Key words:

Hematology. Dairy cows. Postpartum. Cortisol.

\section{Referências}

1 FOSBERG N. Recent insights into ruminant immune function: effects of stress and of immunostimulatory feed products. In: FLORIDA RUMINANT NUTRITION SYMPOSIUM, 2004, Gainesville, FL. Proceedings... [s.I.: s.n.], 2004. p. 81-92.

2 JAIN, N. C. Essentials of veterinary hematology. Philadelphia: Lea \& Febiger, 1993. 417 p.

3 LONGO, L. D. Maternal blood volume and cardiac output during pregnancy: a hypothesis of endocrinologist control. American Journal of Physiology, v. 245, n. 5, p. 720-729, 1983.

4 HYTTEN, F. Blood volume changes in normal pregnancy. Clinics in Hematology, v. 14, n. 3, p. 601612, 1985.

5 TAYLOR, J. A. Leukocyte response in ruminants. In:
FELDMAN, B. F.; ZINKL, J. G; JAIN, N. C. Schalm's veterinary hematology. 5. ed. Philadelphia: Lippincott Williams \& Wilkins, 2000. p. 391-404.

6 ESIEVO, K. A.; MOORE, W. E. Effects of dietary protein and stage of lactation on the hematology and erythrocyte enzymes activities of high-producing dairy cattle. Research in Veterinary Science, v. 26, n. 1, p. 53-58, 1979.

7 ANOSA, V. O.; OGBOGU, D. A. The effect of parturition on the blood picture of sheep. Research in Veterinary Science, v. 26, n. 3, p. 380-382, 1979.

8 MANSTON, R.; RUSSELL, A. M.; DEW, S. M.; PAYNE, J. M. The influence of dietary protein upon blood composition in dairy cows. Veterinary Record, v. 96, n. 23, p. 497-502, 1975.

9 SUZUKI, K.; HIROSE, H.; HOKAO, R.; TAKEMURA, N.; MOTOYOSHI, S. Changes of plasma osmotic pressure during lactation in rats. Journal of Veterinary 
Medical Science, v. 55, n. 4, p. 561-564, 1993.

10 GOFF, J. P.; HORST, R. L. Physiological changes at parturition and their relationship to metabolic disorders. Journal of Dairy Science, v. 80, p. 12601268, 1997.

11 INGVARTSEN, K. L.; DEWHURST, R. J.; FRIGGENS, N. C. On the relationship between lactational performance and health: is it yield or metabolic imbalance that cause production diseases in dairy cattle? A position paper. Livestock Production Science, v. 83 , n. 2-3, p. 277-308, 2003.

12 JACOB, S. K.; RAMNATH, V.; PHILOMINA, P. T.; RAGHUNANDHANAN, K. V.; KANNAN, A. Assessment of physiological stress in periparturient cows and neonatal calves. Indian Journal of Physiology and Pharmacology, v. 45, n. 2, p. 233-238, 2001.

13 BURTON, J. L.; ERSKINE, R. J. Immunity and mastitis. Some new ideas for an old disease. The Veterinary Clinics of North America - food and animal practice, v. 19, p. 1-45, 2003.

14 PAAPE, M.; MEHRZAD, J.; ZHAO, X.; DETILLEUX, J.; BURVENICH, C. Defense of the bovine mammary gland by polymorphonuclear neutrophil leukocytes. Journal of Mammary Gland Biology and Neoplasia, v. 7, p. 109-121, 2002.

15 KIMURA, K.; GOFF, J. P.; KEHRLI JR., M. E.; HARP, J. A. Phenotype analysis of peripheral blood mononuclear cells in periparturient dairy cows. Journal of Dairy Science, v. 82, p. 315-319, 1999.

16 TIZARD, I. R. Veterinary immunology: an introduction. 6. ed. New York: W. B. Saunders, 2000. $482 \mathrm{p}$.

17 VANDEHAAR, M. J.; BUCHOLTZ, H. F.; ALLEN, M. S.; BLACK, J. R.; EMERY, R. S.; SNIFFEN, C. J.; BEVERLY, R. W. Spartan ration evaluator/balancer for dairy cattle. Version 2.01. East Lansing: Michigan State University, 1992.

18 SAS. STATISTICAL ANALISYS SYSTEM. User's guide: statistics. Version. 8.02. Cary, NC: SAS, 2001. 254 p.

19 ABT, D. A.; IPSEN, J.; HARE, W. C. D.; MARSHAK, R. R.; SAHL, J. Circadian and seasonal variations in the hemogram of mature dairy cattle. Cornell Veterinarian, v. 56, p. 479-520, 1966.
20 ANDRESEN, H. A. Evaluation of leukopenia in cattle. Journal of American Veterinary Medical Association, v. 156, n. 7, p. 858-866, 1970.

21 WEISS, D. J.; PERMAN, V. Assessment of the hematopoietic system in ruminants. Veterinary Clinics of North America - food animal practice, v. 8, n. 2, p. 411-428, 1992.

22 LEE, E. K.; KEHRLI JR, M. E. Expression of adhesion molecules on neutrophils of periparturient cows and neonatal calves. American Journal of Veterinary Research, v. 59, p. 37-43, 1998.

23 KEHRLI JR, M. E.; NONNECKE, B. J.; ROTH, J. A. Alterations in bovine neutrophil function during the periparturient period. American Journal of Veterinary Research, v. 50, p. 207-214, 1989.

24 INGVARTSEN, K. L.; ANDERSEN, J. B. Integration of metabolism and intake regulation: a review focusing on periparturient animals. Journal of Dairy Science, v. 83, p. 1573-1597, 2000.

25 REIST, M.; ERDIN, D. K.; VONEUW, D.; TSCHÜMPERLIN, K. M.; LEUENBERGER, H.; CHILLIARD, Y.; HAMMON, H. M.; MOREL, C.; PHILIPONA, C.; ZBINDEN, Y.; KUENZI, N.; BLUM, J. Estimation of energy balance at the individual and herd level using blood and milk traits in high-yielding dairy cows. Journal of Dairy Science, v. 85, p. 3314$3327,2002$.

26 CAI, T. Q.; WESTON, P. G.; LUND, L. A.; BRODIE, B.; MCKENNA, D. J.; WAGNER, W. C. Association between neutrophil functions and periparturient disorders in cows. American Journal of Veterinary Research, v. 55, p. 934-943, 1994.

27 SHUSTER, D. E.; LEE, E. K.; KEHRLI JR., M. E. Bacterial growth, inflammatory cytokine production, and neutrophil recruitment during coliform mastitis in cows within ten days after calving, compared with cows at midlactation. American Journal of Veterinary Research, v. 57, p. 1569-1575, 1996.

28 COELHO, K. O. Impacto dos eventos ocorridos antes e após o parto sobre o desempenho produtivo e reprodutivo na lactação atual e na posterior de vacas holandesas. 2004. 70 p. Dissertação (Mestrado) - Escola Superior de Agricultura "Luiz de Queiroz", Universidade de São Paulo, Piracicaba, 2004. 